\title{
Scenario modeling of hydrodynamics and impurity propagation in Lake Baikal
}

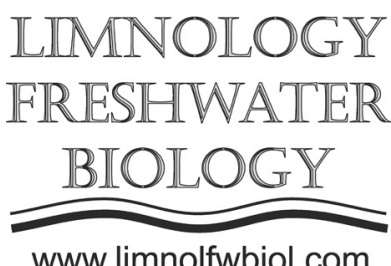

www.limnolfwbiol.com

\begin{abstract}
Tsvetova E.A.*
Institute of Computational Mathematics and Mathematical Geophysics, Siberian Branch of the Russian Academy of Sciences, Pr. Lavrentieva, 6, Novosibirsk, 630090, Russia

ABSTRACT. Some results of scenario calculations of hydrodynamics and the propagation of passive impurities in the lake are presented. We are talking about hypothetical scenarios for the development of lake pollution processes during one summer tourist season. Depending on the chosen scenario, sources of impurities are specified in certain places on the shores of the lake. This simulates the location of tourist sites. To increase the generality, the so-called "climatic" scenarios of atmospheric influences are considered, when data on wind fields and their operational characteristics reflect typical weather conditions in the region. The results of calculations in different scenarios are compared. Their analysis indicates that the functioning of tourism facilities should be regulated taking into account the characteristic features of the lake.
\end{abstract}

Keywords: Mathematical modeling, hydrodynamics, distribution of impurities, scenario approach

\section{Introduction}

The purpose of mathematical modeling of processes and phenomena occurring in natural objects is to obtain new knowledge about these objects and processes. Certainly, the basic knowledge of Lake Baikal is knowledge of the system of currents, against which all chemical, biological, environmental and other processes develop. In the 70s of the last century, the author's work was one of the first to study the hydrodynamics of the lake using mathematical modeling methods (Tsvetova, 1977). Since then, models have occupied their niche in Baikal studies due to efforts of Russian and foreign scientists (see the review by Ovchinnikova and Bocharov, 2017).

Features of modeling natural processes are the presence of a large number of uncertainties in the formulation of models, in setting parameters. Despite the long history of studying the lake, not everything is clear in the cause-and-effect relationship, especially in interdisciplinary research. Methods of computer implementation also make their own requirements. In modern models with the necessary description of geometric scales, a large number of parameters are required. In practice, it turns out that there is always not enough data for adequate reproduction of processes.

We use a scenario approach, which in this situation is the main way to achieve results. It allows you to get a variant assessment of the results depending on the specified parameters.

\section{Material and methods}

To date, the author has created a complex of mathematical models of various degrees of complexity for studying processes on the large and local scales. For its implementation, algorithms, programs and modeling technology have been developed. Until recently, the basic model of the hydrodynamics was a three-dimensional non-stationary model in the nonhydrostatic approximation; its first version is presented in (Tsvetova, 1999). Since hydrodynamics is responsible for the distribution of impurities that pollute the lake, a necessary complement to the complex is a set of impurity distribution models.

With the discovery of methane hydrates at the bottom of the lake, new problem statements and, consequently, new model formulations have emerged (Tsvetova, 2015). In particular, a mathematical model of a multiphase system has been developed, which is formulated in the so-called Euler-Euler statement (Nigmatulin, 1990). In it, the phases are assumed to be interpenetrating continua. It is believed that water is the main carrier medium.

\section{Results and discussion}

To demonstrate the capabilities of models and scenarios, the report presents the results of calculations with various options for specifying sources of pollution associated with the development of tourism on Lake 
Baikal. The simulation is based on a three-dimensional non-stationary non-hydrostatic model of the lake. We are talking about hypothetical scenarios for the development of lake pollution processes during one summer tourist season. To increase generality, it is not a specific year and season that is considered, but the so-called "climatic" scenarios of atmospheric influences, when data on wind fields and their operational characteristics are set in accordance with the classification from (Atlas, 1977). Using these data in the Monte Carlo method, atmospheric impact scenarios are compiled. In turn, atmospheric scenarios are taken into account in boundary conditions on the surface of the lake. Together with the calculated thermal regime of the lake, taking into account the seasonal and intraday variability of the heat balance on the surface, they are responsible for the formation of the current system. Simultaneously with the system of currents, propagation of impurities are reproduced. In this case, the impurities are considered as passive tracers. The sources of impurities in the scenarios are hypothetical tourist sites located on the shores of the lake.

Impurity propagation results are compared in various scenarios. Their analysis suggests that the functioning of tourist facilities should be regulated when assessing and planning the development of the industry in order to preserve the lake as a World Heritage Site.

\section{Conclusion}

In conditions of increased load on the ecosystem of the lake, which has already led to a change in the species composition of its inhabitants (Kravtsova et al., 2020), there is an urgent need for regulatory measures on the part of the competent authorities. Quantitative information, even in hypothetical scenarios such as those presented in the report, may be useful. Mathematical modeling, coupled with the scenario approach, just provides a working tool for evaluations, but it needs data to adjust to the real state of the lake system.

\section{Acknowledgments.}

Regarding the development of basic mathematical models, the work is carried out within the framework of the state task of the ICMMG SB RAS No. 0315-20190004. The development of algorithms is supported by the RFBR project No. 20-01-00560.

\section{References}

Atlas volneniya i vetra oz. Baikal [Atlas of waves and winds of Lake Baikal]. 1977. In: Rzeplinski G.V., Sorokina A.I. (Eds.). Leningrad: Gidrometeoizdat. (in Russian)

Kravtsova L.S., Mizandrontsev I.B., Vorobyova S.S. et al. 2020. Influence of water motion on the spatial distribution of Spirogira in Lake Baikal. Journal of Great Lakes Research 46: 29-40. DOI: 101016/j.jglr.2019.09.004

Nigmatulin R.I. 1990. Dynamics of multiphase media: v. 1-2. New York: Hemisphere Publishing Corporation.

Ovchinnikova T.E.; Bocharov O.B. 2017. Numerical simulation of water exchange processes in Lake Baikal. Water Resources 44: 453-462. DOI: 10.1134/S0097807817030150

Tsvetova E.A. 1977. Mathematical modeling of Lake Baikal water circulation. In: Afanasiev A.N., Verbolov V.I. (Eds.), Techeniya $\mathrm{v}$ Baikale [Currents in Lake Baikal]. Novosibirsk, pp. 63-84. (in Russian)

Tsvetova E.A. 1999. Mathematical modelling of Lake Baikal hydrodynamics. Hydrobiologia 407: 37-43. DOI: 10.1023/A:1003766220781

Tsvetova E.A. 2015. Modeling of hydrodynamics of water-methane heterogeneous system. Proceedings of SPIE the International Society for Optical Engineering 9680. DOI: $10.1117 / 12.2205998$ 